\title{
Extending the wavelength range of multi-spectral microscope systems with Fourier ptychography
}

Antony Chi Shing Chan, Cheng Shen, Elliott Williams, Xiaoyu Lyu, Hangwen Lu, et al.

Antony Chi Shing Chan, Cheng Shen, Elliott Williams, Xiaoyu Lyu, Hangwen Lu, Craig Ives, Ali Hajimiri, Changhuei Yang, "Extending the wavelength range of multi-spectral microscope systems with Fourier ptychography," Proc. SPIE 10890, Label-free Biomedical Imaging and Sensing (LBIS) 2019, 1089020 (25 March 2019); doi: 10.1117/12.2510875

SPIE. Event: SPIE BiOS, 2019, San Francisco, California, United States 


\section{Extending the wavelength range of multi-spectral microscope systems with Fourier ptychography \\ SPIE. \\ Antony Chi Shing Chan ${ }^{1}$, Cheng Shen ${ }^{1}$, Elliott Williams ${ }^{1}$, Xiaoyu Lyu², Hangwen Lu ${ }^{3}$, Craig Ives ${ }^{1}$, \\ Ali Hajimiri ${ }^{1}$ and Changhuei Yang ${ }^{1 *}$ \\ ${ }^{1}$ Department of Electrical Engineering, Caltech (United States) ${ }^{2}$ Academy of Opto-Electronics, CAS (China) ${ }^{3}$ Google LLC}

\section{Introduction}

Limited by manufacturing and optical materials, even the best designed multi-spectral objective will exhibit residual monochromatic aberration and chromatic aberration. Here we propose a multi-spectral imaging scheme based on the Fourier Ptychographic Microscopy (FPM), which can render in-focus images by digital aberration correction. Also, the wavelength extension to Near Infrared (NIR) regime broadens the application of our system, like imaging through bonded silicon wafer devices for locating defects.

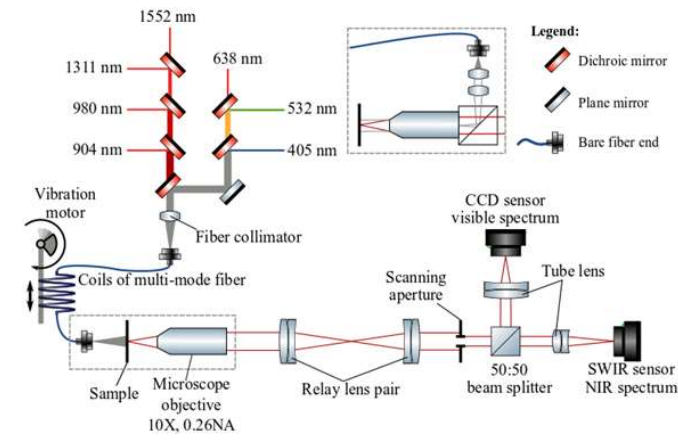

Fig. 1 Schematic of multi-spectral Fourier ptychographic microscope, including transmission and reflection mode.

\section{Aberration Characterization}

- Principle

$$
\begin{gathered}
\underset{w_{i}}{\arg \min } L\left(\tilde{B}_{\text {on-axis }}(u, v) \cdot e^{i 2 \pi z(u, v)}-\tilde{B}_{\text {off-axis }}(u, v)\right) \\
z(u, v)=\sum_{i=1}^{8} w_{i} Z_{i}(u, v)
\end{gathered}
$$

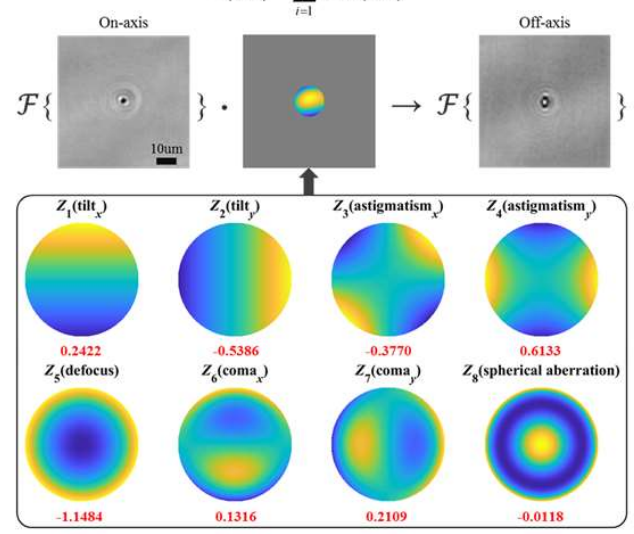

Fig. 2 Digital aberration calibration based on Zernike mode decomposition, taking the complex amplitude of an on-axis microbead as reference.

\section{Aberration Characterization}

\section{- Result}

For an objective with a low NA but a large field-of-view (FOV), its aberration is spatial variant Usually, aberrations increase as a function of distance from the image center.

$$
w_{i}(x, y), i=1, \cdots, 8
$$
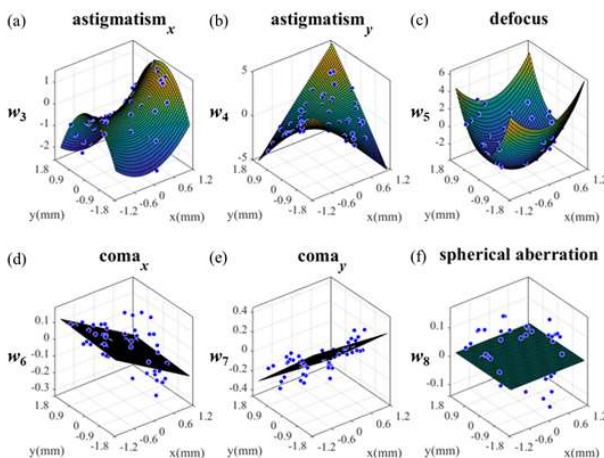

Fig. 3 Spatially varying aberration characterization for th whole FOV under the wavelength of $532 \mathrm{~nm}$. (a)-(f) fitted 2D surface for each type of aberration (first order Zernike modes are normally not considered as aberrations, thus not shown).

\section{Digital Aberration Correction}

Since the location-dependent aberration composed of different Zernike modes has been calibrated first, digital aberration correction can be done for other samples to obtain the clear and undistorted images.

$$
\text { 1. A large FOV }\left(\sim 1.92 \times 1.53 \mathrm{~mm}^{2}\right)
$$

2. A better way of pixel super-resolution
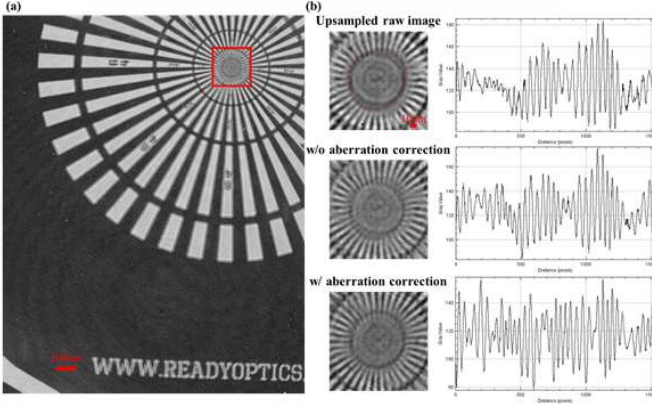

Fig. 4 Demonstration of digital aberration characterization under the wavelength of $1552 \mathrm{~nm}$. (a) the whole FOV raw data. (b) Comparison between the pixel upsampling result and FPM reconstruction without/with aberration correction.

\section{Imaging of Silicon Chip}

With our system, the non-invasive detection on the silicon wafer devices, like flip-chips, is possible. Usually, the frontside imaging could directly visualize the top metallic layers, but the ones deep inside can only be detected from the backside with the aid of NIR light thanks to the fairly low absorption of silicon to this regime. Besides, FPM reconstruction can further enhance the imaging resolution and digitally refocus to different components beneath.

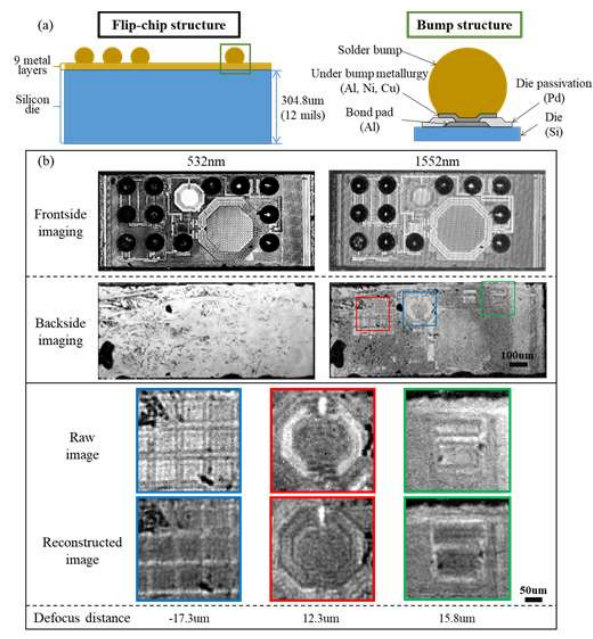

Fig. 5 Imaging of a flip-chip under reflection mode. (a) the schematic of the flip-chip. (b)-(c) the front and back side imaging of the flip-chip. The bottom metal layers could only be visible under NIR light. Different components are displayed in the enlargements.

\section{Conclusions}

Our multi-spectral FPM system enables:

1. Digital aberration correction

2. Multi-spectral high-resolution imaging with large FOV 3. Digital refocusing

4. Non-invasive defect detection of silicon wafer devices

\section{References}

\footnotetext{
1. Zheng, G., Ou, X., Horstmeyer, R., \& Yang, C. (2013). Characterization of spatially varying aberrations for wide field-o view microscopy. Optics express, 21(13), 15131-15143.

2. Zheng, G., Horstmeyer, R., \& Yang, C. (2013). Wide-field, highresolution Fourier ptychographic microscopy. Nature photonics, 7(9), 739-745.

3. Ou, X., Chung, J., Horstmeyer, R., \& Yang, C. (2016). Aperture scanning Fourier ptychographic microscopy. Biomedical optics express, $7(8), 3140-3150$
} 